\title{
Modeling and Simulation of a Synchronous Generator with Rotor Angle Stability and Solve Inter Area Mode of Oscillation in Power System using Power System Stabilizer(PSS)
}

\author{
Md. Abu Hena Shatil and Md. Lutfur Rahman
}

\begin{abstract}
Power System stabilizers are a form of supplementary control that is used to provide additional damping to the inter area mode oscillations or to stabilize a generator whose voltage regulator gain is such that it may result in negatively damped machine-to-system oscillations under certain conditions. It has seen observed that the damping of these small power oscillations can be improved by leading back appropriate stabilizing signals to the input of the gain's exciter. Some input signals that have been considered in the research are slip speed, accelerating power, frequency. In this manuscript, we will use an establish approach to obtain a preliminary design for a power system stabilizer with slip speed as the feedback signal.
\end{abstract}

Keywords-Power system stabilizer, Generator, Exciter, Interarea oscillation, Rotor angle stability.

\section{INTRODUCTION}

Neoteric power system is a complicated network comprising of many generators, variety of loads, transmission lines and transformers. As a consequence of increasing power demand various transmission lines are more loaded than was planned when they are built. Interconnections of the power system are of extreme convenience on the other hand it also brings lot of new problems such as low frequency oscillations. For that the transmission capacity decreased [1]-[2].Low frequency oscillations consist of inter area modes and local modes, which are associated with local generators and generators in different areas. Power system stabilizer can make the system more stable [3]-[4]. The main function of a power system stabilizer is to add damping to generator rotor oscillations by controlling its excitation using auxiliary stabilizing signal. To provide damping the power system stabilizer must produce a component of electrical torque in phase with rotor speed deviation [5]-[6].

Md. Lutfur Rahman completed Master of Science in Electrical and Electronic Enguneering (MEEE) from American International University-Bangladesh (AIUB), Kuril, Dhaka-1229, Bangladesh. Email:

md.lutfurrahmanaiub@gmail.com

Md. Abu Hena Shatil is an Assistant Professor of the department of Electrical and Electronic Enguneering (EEE), American International UniversityBangladesh (AIUB), Kuril, Dhaka-1229, Bangladesh.. Email: abu.shatil@aiub.edu
The various modes of oscillation can be grouped into three broad categories. First, Intra plant modes in which only the generators in a power plant participate. For Intra plant modes oscillation frequencies are generally very high in the range of 1.5 to $3.0 \mathrm{~Hz}$. Second, Local modes in which several generators in an area participate. For Local modes frequencies of oscillations are in the range of 0.8 to $1.8 \mathrm{~Hz}$. Third one Inter area mode in which generators over an extensive area participate. For Inter area mode oscillations frequencies are low and in the range of 0.2 to $0.5 \mathrm{~Hz}$. Inter-area oscillations are inherent to power systems, should these oscillations are poorly damped the power interchange between the power system areas may be limited for security reasons [7], [8]. Number of factors are responsible for Inter-area oscillations [9] and system loading condition is one of them. Today's most of the power systems worldwide are forced to operate closer to their security limits due to environmental and economic constraints [10], [11].

It has been indicated that inter-area mode oscillations are a major constraint for power transfer increase between regions in a power system, predominantly between weakly interconnected areas during high loading conditions [7], [8]. An example of this type of problems, occurred in a real power system and caused the system blackout, is reported in reference [12].

In this paper discussed the performance of power system stabilizer for damping the inter area oscillations of inter area power system. In section II \& III, system description, modeling and simulation are presented. In Section IV, simulation results are presented.

\section{SYSTEM DISCRIPTION}

\section{A. Selecting Synchronous generator and control}

The synchronous generator model mainly used in power system studies vary from the easier electromechanical model to more complex ones with different types of impedance parameters and the time constants included to capture more appropriate dynamics of the system Inter-area mode has been used in the study. The generating units mainly consists of an excitation control system which represented by IEEE Type 1 DC Exciter. 
For this system used a synchronous generator model. Which represent as Tmodel. That Tmodel contains generator part of the rotor and stator. For control generator part used some controller. For designing purpose simulation tool used.

\section{B. Rotor angle stability}

Rotor angle stability: This is generator driven and it measures the ability of generators in the interconnected power system to remain synchronous after a system disturbance. Type of disturbance rotor-angle stability consideration can be further classified into small signal stability or steady-state stability, and large disturbance stability or transient stability. It is dependent on the capacity of machine to maintain the equilibrium state in between electro-magnetic torque and mechanical torque of each synchronous machine in the system [13].

Small Signal Stability: The capacity of power system to retain the synchronism state when subjected under small disturbances is known as small signal stability [14]. Instability may be of two forms (i) Scarcity of synchronizing torque which leads to steady increase in generator-rotor angle. (ii) Lack of adequate torque due to which rotor oscillations with increasing amplitude are generated. At present time, the problem of small signal stability occur due to inadequate damping of oscillations.

Transient Stability: The capacity of power system of returning towards the normal operating condition after facing severe disturbances namely single phase or multi-phase short circuit or the loss of generator is known as transient stability [13].

\section{Inter area mode osillation}

Oscillation: The variations in the value of voltage above and below some mean value in an alternating current is called as Oscillation. These oscillations cause instability in a power system. And if they do not damped effectively, they may cause the breaking of entire power system.

Interarea mode oscillations: This system is observed over a large part of the network. which involves two coherent groups of generators swinging against each other at $1 \mathrm{~Hz}$ or may be less. The variation in tie-line power can be large which shown in Figure 01.

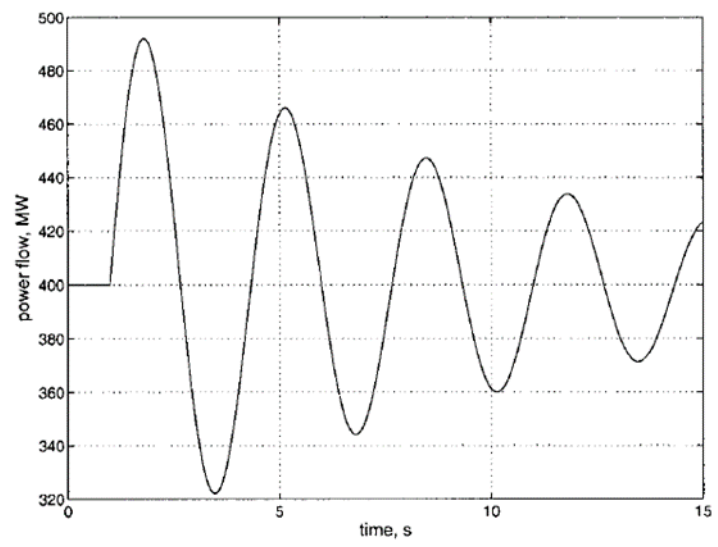

Fig. 1. A typical instance of inter-area mode oscillation [3]
The oscillation frequency is approximately $0.3 \mathrm{~Hz}$. This complex phenomenon involves many parts of the system with highly non-linear dynamic behavior. The damping characteristic of the interarea mode is dictated by the tie-line strength, the power flow through the interconnection and the nature of the loads and the interaction of loads with the dynamics of generators and their associated controls. The operation of the system in the presence of a lightly damped interarea mode is very difficult [14].

\section{MODELLING AND SIMULATION}

\section{A. Small signal repressantaion system}

Figure 02 shows the Small-signal representation of system of transfer function blocks of generator with exciter and pss. Figure 03 shows the type 1 exciter.

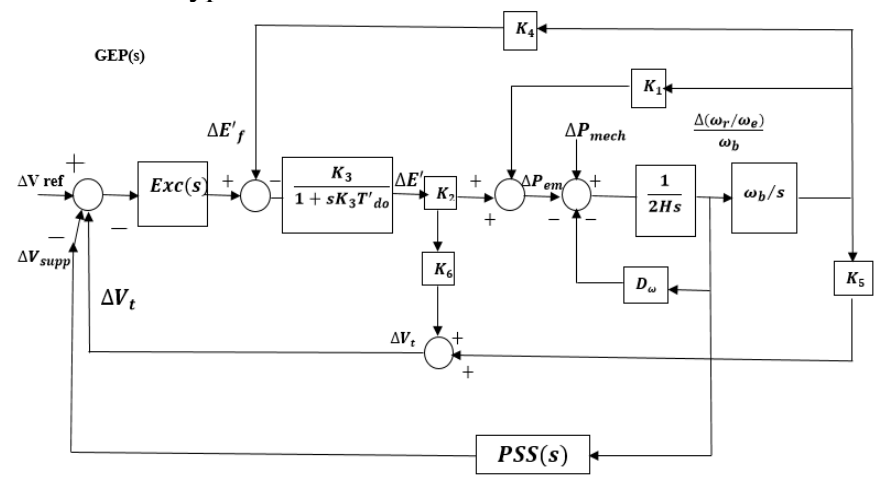

Fig. 2. Small-signal representation of system of transfer function blocks of generator with exciter and pss using slip speed [4]

Following the design firstly we will determine the transfer function, PSS(s), of a power system stabilizer with slip speed as its input signal. Starting with the condition that the resulting torque component of $\Delta P_{e m}$ produced by the power system stabilizer (pss) modulation be 180 degrees out of phase with $\Delta \omega_{r}-\omega_{e} / \omega_{b}$ as in the case of the damping torque from $D_{\omega}$, we have

$\Delta P_{e m}=-G E P(s) P S S(s) \Delta\left(\frac{\omega_{r}-\omega_{e}}{\omega_{b}}\right)$

$\frac{\Delta P_{e m}}{\Delta\left(\frac{\omega_{r}-\omega_{e}}{\omega_{b}}\right)}=-G E P(s) P S S(s)=-D_{p s s}$

here $\Delta P_{e m}$ is the perturbation component of the electromagnetic power that is produced by the power system stabilizer(pss) modulation signal, GEP(s) is the transfer characteristic of the generator and excitation system to the modulation signal, PSS(s) is the transfer function of the power system stabilizer and $D_{p s s}$ is a positive coefficient. For the negative sign on the right side of Equation 2 taken care of by inverting the sign of the $v_{\text {supp }}$ input for the excitation system, which shown in Figure 3 . 


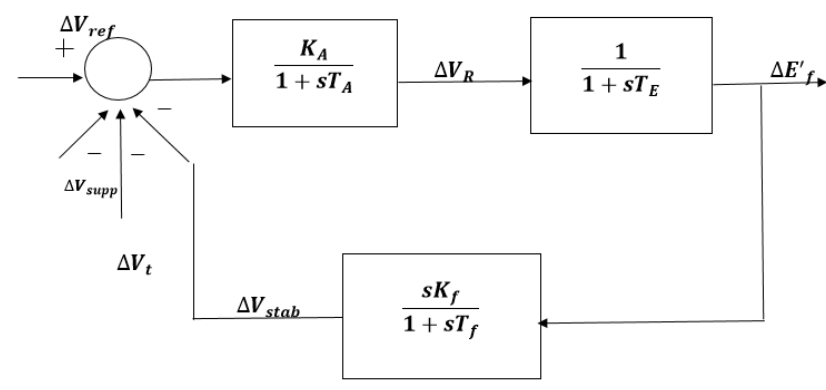

Figure 03: Small-signal representation of system of transfer function of the exciter [5]

Now an expression for the GEP(s) can be define by ignoring the contributions by $K_{4}$ and $K_{5}$ to the shady blocks in Figure 02, assuming that the speed deviation is small.

$G E P(s) \approx K_{2} \frac{E x c(s) \frac{K_{3}}{1+s K_{3} T^{\prime} d o}}{1+K_{6} \operatorname{Exc}(s) \frac{K_{3}}{1+s K_{3} T^{\prime} d o}}$

Figure 03 shown the excitation system of the transfer function, $\operatorname{Exc}(\mathrm{s})$, is

$\operatorname{Exc}(s)=\frac{\Delta E_{f}}{\Delta V_{t}}=\frac{\frac{K_{A}}{1+s T^{1+s T_{E}}}}{1+\frac{K_{A} 1}{1+s T_{A} A^{1+s T_{E}} E^{1+s T_{f}}}}$

Figure 08 shows the Bode plot of the exciter using the set parameters.

\section{B. Linearzied model of generator and network}

In this section, we begin by deriving linearized equation of the system retaining the identity of variables $\Delta V, \Delta E^{\prime}{ }_{q} \Delta E_{f}$ and $\Delta \delta$. The linear model so derived is being used in $\mathrm{m}$-file to determine certain transfer functions for the preliminary design of the pss.

The usually small stator winding resistance, $r_{s}$, will be neglected in this derivation. In per unit, the generated power, $p_{e m}$, may be expressed as $E_{q o} i_{q}$ or $E_{q}^{\prime} i_{q}+\left(x_{q}-x_{d}^{\prime}\right) i_{d} i_{q}$ Taking small displacements about the steady-state operating values denoted by an additional subscript o, we will obtain

$$
\begin{aligned}
\Delta P_{e m} & =E_{q o} \Delta i_{q}+i_{q o} \Delta E_{q} \\
& =E_{q o} \Delta i_{q}+i_{q o}\left(\Delta v_{q}+x_{q} \Delta i_{d}\right) \\
& =E_{q o} \Delta i_{q}+i_{q o}\left\{\Delta E^{\prime}{ }_{q}+\left(x_{q}-x^{\prime}{ }_{d}\right) \Delta i_{d}\right\}
\end{aligned}
$$

Taking small displacements expression for the generator's terminal voltage magnitude, $V_{t}^{2}=v_{q}^{2}+v_{d}^{2}$ and using the relations $E_{q}^{\prime}=v_{q}+x_{d}^{\prime} i_{d}$ and $v_{d}=x_{q} i_{q}$ we obtain $2 V_{t} \Delta V_{t}=2 v_{q o} \Delta v_{q}+2 v_{d o} \Delta v_{d}$

$$
\Delta V_{t}=\frac{v_{q o}}{V_{t}}\left(\Delta E_{q}^{\prime}-x_{d}^{\prime} \Delta i_{d}\right)+\frac{v_{d o}}{V_{t}} x_{q} \Delta i_{q}
$$

Taking small displacements of the $f$ rotor winding equation, we obtain
$T^{\prime}{ }_{d o} \frac{d E^{\prime}{ }_{q}}{d t}+\Delta E_{q}^{\prime}=\Delta E_{f}+\left(x_{d}-x_{d}^{\prime}\right) \Delta i_{d}$

Denoting the infinite bus quantities by an additional subscript, $i$, the $q d$ voltage equations of the infinite bus in the generators rotor reference frame are

$v_{q i}=E_{q}^{\prime}-\left(x_{d}^{\prime}-x_{e}\right) i_{d}-r_{e} i_{q}$

$v_{d i}=\left(x_{q}+x_{e}\right) i_{q}-r_{e} i_{d}$

When infinite bus voltage magnitude, $V_{i}$, the rotor angle, $\delta$, we have

$$
v_{q i}-j v_{d i}=V_{i} e^{-j \delta}=V_{i}(\cos \delta-j \sin \delta)
$$

Solving Equation 8 for $i_{q i}$ and $i_{d i}$, we obtain

$i_{q}=\frac{r_{e}}{D_{Z}}\left(E_{q}^{\prime}-V_{i} \cos \delta\right)+\frac{\left(x_{e}+x_{d}^{\prime}\right)}{D_{Z}} V_{i} \sin \delta$

$i_{d}=\frac{\left(x_{e}+x_{q}\right)}{D_{Z}}\left(E^{\prime}{ }_{q}-V_{i} \cos \delta\right)-\frac{r_{e}}{D_{Z}} V_{i} \sin \delta$

Where $D_{z}=r_{e}^{2}+\left(x_{e}+x_{q}\right)\left(x_{e}+x_{d}^{\prime}\right)$ assuming that the infinite bus voltage, $V_{i}$, is constant and taking the small displacements of Equation 10, we get

$\Delta i_{q}=\frac{r_{e}}{D_{Z}} \Delta E^{\prime}{ }_{q}+\frac{V_{i}}{D_{Z}}\left\{r_{e} \sin \delta_{o}+\left(x_{e}+x^{\prime}{ }_{d}\right) \cos \delta_{o}\right\} \Delta \delta$

$\Delta i_{d}=\frac{\left(x_{e}+x_{q}\right)}{D_{Z}} \Delta E^{\prime}{ }_{q}-\frac{V_{i}}{D_{Z}}\left\{r_{e} \cos \delta_{o}-\left(x_{e}+x^{\prime}{ }_{d}\right) \sin \delta_{o}\right\} \Delta \delta$

Using the expression equation 11 to replace qd current terms in equation 5 and regrouping the $\Delta \delta$ and $\Delta E^{\prime}{ }_{q}$ terms, Equation 5 can be written as

$\Delta P_{e m}=K_{1} \Delta \delta+K_{2} \Delta E_{q}^{\prime}$

Where,

$$
\begin{aligned}
K_{1}=\frac{E_{q o} V_{i}}{D_{Z}}\{ & \left.r_{e} \sin \delta_{o}+\left(x_{e}+x^{\prime}{ }_{d}\right) \cos \delta_{o}\right\}+ \\
& \frac{i_{q o}\left(x_{q}+x^{\prime}{ }_{d}\right) V_{i}}{D_{Z}}\left\{\left(x_{e}+x_{q}\right) \sin \delta_{o}-r_{e} \cos \delta_{o}\right\}
\end{aligned}
$$

$K_{2}=\frac{E_{q o} r_{e}}{D_{Z}}+\frac{i_{q o}}{D_{Z}}\left\{1+\left(x_{q}-x_{d}^{\prime}\right)\left(x_{e}+x_{q}\right)\right\}$

Similarly, replacing the $q d$ current terms in equation 6 and 7 and regrouping the $\Delta \delta$ and $\Delta E^{\prime}{ }_{q}$ trems, we can express the small displacements equations of the stator terminal voltage and rotor $f$ winding as

$T^{\prime}{ }_{d o} \frac{d \Delta E^{\prime} q}{d t}+\frac{\Delta E^{\prime} q}{K_{3}}=\Delta E_{f}-K_{4} \Delta \delta$

$\Delta V_{t}=K_{5} \Delta \delta+K_{6} \Delta E_{q}^{\prime}$

Where,

$K_{3}=\left\{1+\frac{\left(x_{d}-x_{d}^{\prime}\right)\left(x_{e}+x_{q}\right)}{D_{Z}}\right\}^{-1}$ 
$K_{4}=\frac{V_{i}\left(x_{d}-x_{d}^{\prime}\right)}{D_{Z}}\left\{\left(x_{e}+x_{q}\right) \sin \delta_{o}-r_{e} \cos \delta_{o}\right\}$

$K_{5}=V_{i} \frac{x_{d o}}{V_{t}} \frac{x_{q}}{D_{Z}}\left\{r_{e} \sin \delta_{o}+\left(x_{e}+x^{\prime}{ }_{d}\right) \cos \delta_{o}\right\}$

$$
+V_{i} \frac{v_{q o}}{V_{t}} \frac{x_{d}^{\prime}}{D_{Z}}\left\{r_{e} \cos \delta_{o}-\left(x_{e}+x_{q}\right) \sin \delta_{o}\right\}
$$

$K_{6}=\frac{v_{q o}}{v_{t}}\left\{1-\frac{x_{d}^{\prime}\left(x_{e}+x_{q}\right)}{D_{Z}}\right\}+\frac{v_{d o}}{v_{t}} \frac{x_{q} r_{e}}{D_{Z}}$

Now taking small displacements for the rotor equations, we will achieve

$\frac{d \Delta\left(\omega_{r} / \omega_{b}\right)}{d t}=\frac{1}{2 H}\left\{\Delta P_{m e c h}+\Delta P_{e m}-D_{\omega}\left(\omega_{r} / \omega_{b}\right)\right\}$

$\frac{d \Delta \delta}{d t}=\omega_{b}\left(\Delta \omega_{r} / \omega_{b}\right)$

Similarly, taking small displacements of the excitation systems equations, will obtain

$\frac{d \Delta E_{f}}{d t}=\frac{1}{T_{E}} \Delta V_{R}$

$\frac{d \Delta V_{R}^{\prime}}{d t}=\frac{K_{A}}{T_{A}}\left(\Delta V_{\text {ref }}-\Delta V_{t}-\Delta V_{\text {stab }}-\Delta V_{\text {supp }}\right)-\frac{\Delta V_{R}}{T_{A}}$

$\frac{d \Delta V_{\text {stab }}}{d t}=\frac{K_{f}}{T_{f} T_{E}}\left(\Delta V_{R}-\Delta E_{f}\right)-\frac{\Delta V_{\text {stab }}}{T_{f}}$

\section{Equations}

Stator Winding equations:

$v_{q}=-r_{s} i_{q}-x^{\prime}{ }_{d} i_{d}+E^{\prime}{ }_{q} \quad p u$

$v_{d}=-r_{s} i_{d}-x^{\prime}{ }_{q} i_{q}+E_{d}^{\prime}$

$E_{q}=E_{q}^{\prime}+\left(x_{q}-x_{d}^{\prime}\right) i_{d}$

Rotor Winding equations:

$T^{\prime}{ }_{d o} \frac{d E^{\prime} q}{d t}+E_{q}^{\prime}=E_{f}+\left(x_{d}-x^{\prime}{ }_{d}\right) i_{d}$

$E_{d}^{\prime}=E_{q}^{\prime}+\left(x_{q}-x_{q}^{\prime}\right) i_{d}$

Torque equations:

$$
T_{e m}=-\left\{E^{\prime}{ }_{q} i_{q}+E_{d}^{\prime} i_{d}+\left(x^{\prime}{ }_{q}-x^{\prime}{ }_{d}\right) i_{d} i_{q}\right\}=-E_{q} i_{q} p u
$$

Rotor equations:

$2 H \frac{d\left\{\frac{\left(\omega_{r}-\omega_{e}\right)}{\omega_{b}}\right\}}{d t}=T_{e m(p u)}+T_{m e c h(p u)}-T_{d a m p(p u)} p u$
$\frac{d \delta_{e}}{d t}=\omega_{r}-\omega_{e}$

\section{Simulations}

TABLE I.

\begin{tabular}{|c|c|c|c|}
\hline $\begin{array}{c}\text { Gen. } \\
\text { Parameter }\end{array}$ & Ratings & $\begin{array}{c}\text { Exc. } \\
\text { Parameter }\end{array}$ & Ratings \\
\hline Rated MVA & 920.35 & Rated kV & 18 \\
\hline Rated P.F. & 0.9 & S.C.R & 0.58 \\
\hline $\begin{array}{ll}x_{d} & \mathrm{Pu}\end{array}$ & 1.790 & $\begin{array}{ll}x_{q} & \mathrm{Pu}\end{array}$ & 1.660 \\
\hline$x_{d}^{\prime}{ }_{d} \mathrm{Pu}$ & 0.355 & $x_{q}^{\prime} \mathrm{Pu}$ & 0.570 \\
\hline$x^{\prime \prime}{ }_{d} \mathrm{Pu}$ & 0.275 & $x_{q}^{\prime \prime} \mathrm{Pu}$ & 0.275 \\
\hline $\begin{array}{ll}x_{l s} & \mathrm{Pu}\end{array}$ & 0.215 & $\begin{array}{ll}r_{s} & \mathrm{Pu}\end{array}$ & 0.0048 \\
\hline$T^{\prime}{ }_{d o} \mathrm{Sec}$ & 7.9 & $T_{q o}^{\prime} \mathrm{sec}$ & 0.41 \\
\hline$T^{\prime \prime}{ }_{\text {do }} \mathrm{Sec}$ & 0.032 & $T_{q o}^{\prime \prime} \mathrm{sec}$ & 0.055 \\
\hline $\mathrm{H} \mathrm{Sec}$ & 3.77 & $D_{\omega} \mathrm{Pu}$ & 2 \\
\hline $\begin{array}{ll}K_{A} & \mathrm{Pu}\end{array}$ & 50 & $T_{A} \mathrm{sec}$ & 0.07 \\
\hline$V_{R}^{\max } \mathrm{pu}$ & 1 & $V_{R}^{\min } \mathrm{Pu}$ & -1 \\
\hline $\begin{array}{ll}K_{E} & \mathrm{Pu}\end{array}$ & -0.0465 & $T_{E} \mathrm{sec}$ & 0.052 \\
\hline$A_{E x}$ & -0.0012 & $B_{E x}$ & 1.264 \\
\hline$K_{f} \mathrm{Pu}$ & 0.0832 & $T_{f} \mathrm{sec}$ & 1.00 \\
\hline
\end{tabular}

PARAMETERS FOR GENERATOR \& EXCITATION

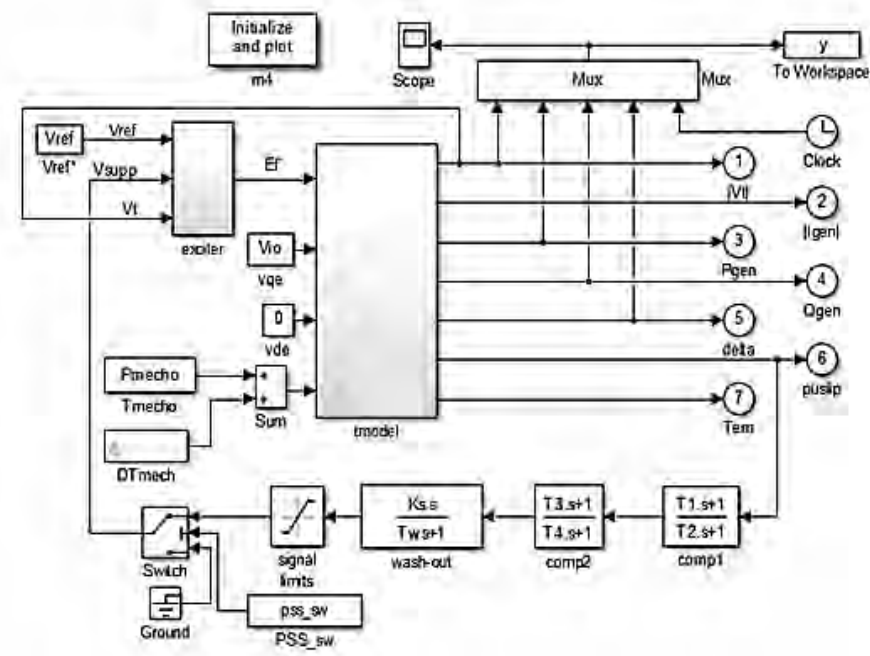

Fig. 3. Overall diagram of synchronous generator with exciter and power system stabilizer 


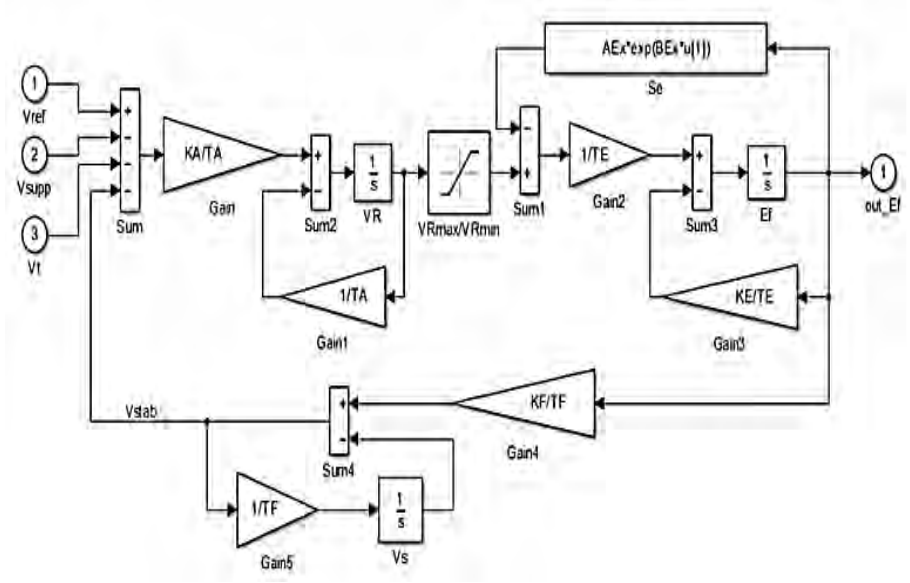

Fig. 4. Simulation of synchronous generator with exciter and power system stabilizer

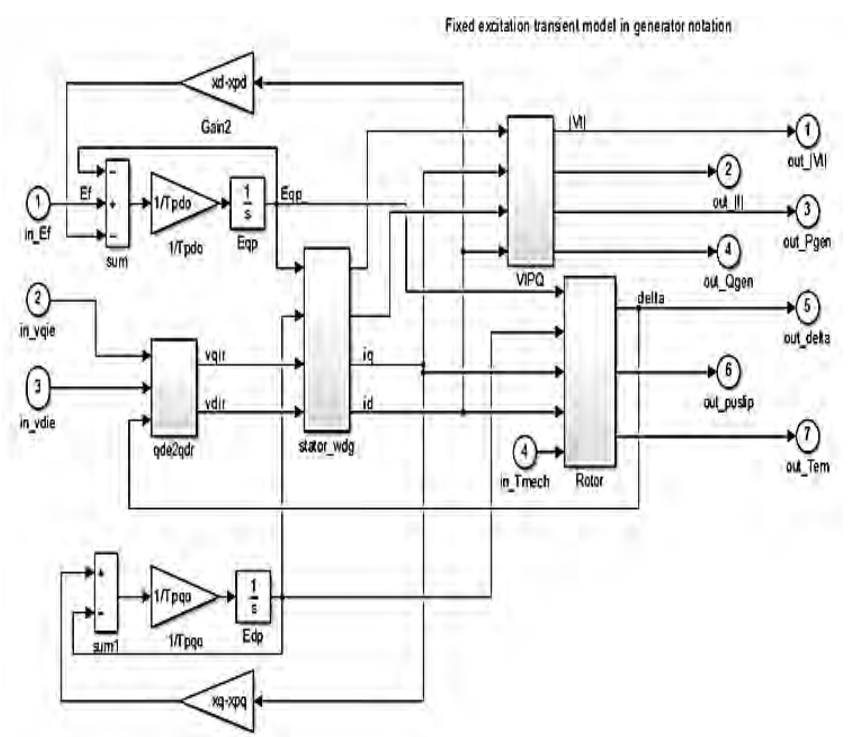

Fig. 5. Simulation of synchronous generator of T-model

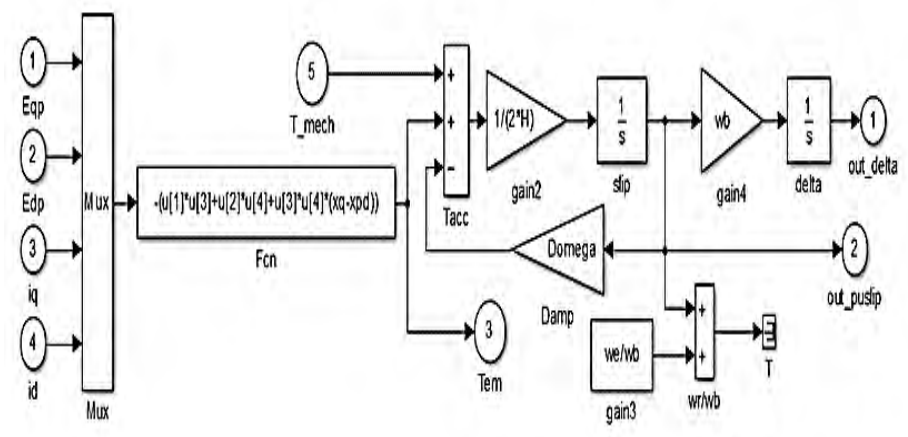

Fig. 6. Simulation of synchronous generator of rotor

Figure 4 shows the simulation of the overall diagram, of a synchronous generator with exciter and pss, that is connected by a series RL line to an infinite bus. The tmodel block contains the same transient model. Figure 05 shows the SIMULINK simulation of the exciter. The supplementary input, is the modulation signal from the pss. Figure 5 shows Simulation of synchronous generator of T-model. Figure 6 shows Simulation of synchronous generator of rotor

\section{SimULATION RESULT ANALYSIS}

Determining GEP (s) and Exc(s):
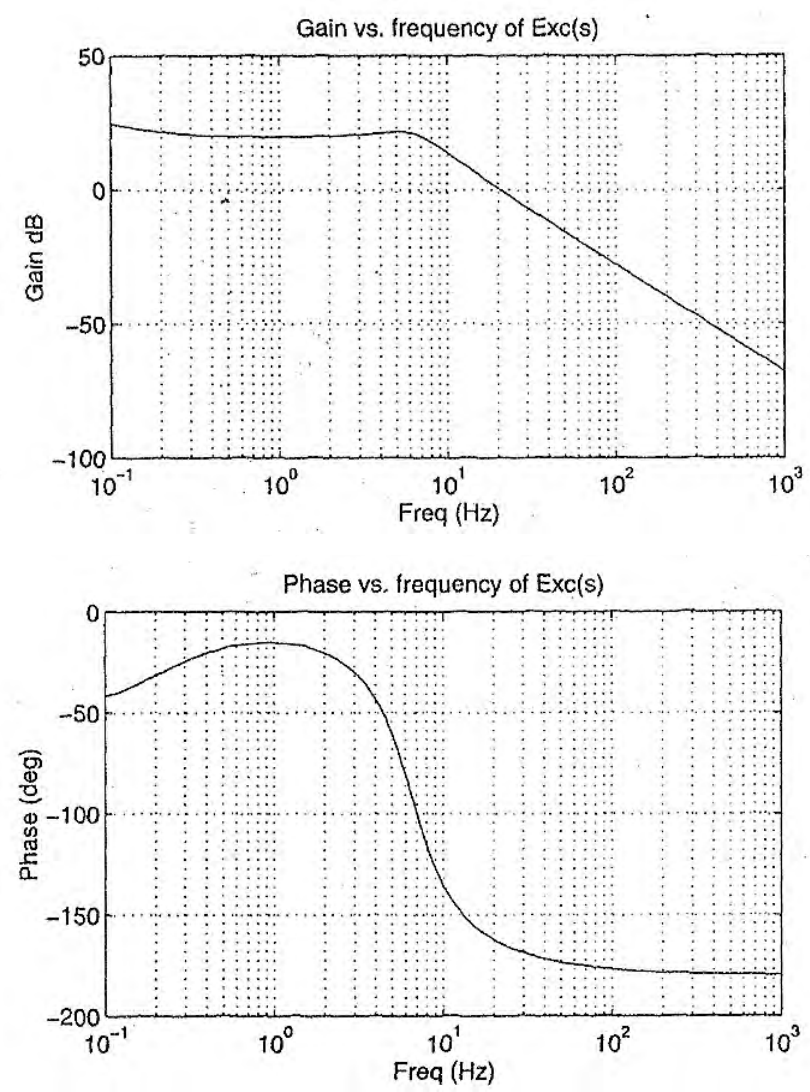

Fig. 7. Bode plot of $\operatorname{Exc}(\mathrm{s})$ using set parameters

For this system, the MATLAB M-file, uses the expressions given in Equation 3 and 4 instead to obtain these two transfer functions. It firstly computes the desired operating point values to set up the transfer functions, GEP(s) and $\operatorname{Exc}(\mathrm{s})$, and then it uses the GEP(s) to determine the transfer function, PSS(s), using Equation 2 Besides determining the transfer functions, $\mathrm{m}$ file provides the root-locus and Bode plots of the transfer functions, initializes the SIMULINK simulation, and sets it up for a small disturbance study to verify the dynamic response. Figures 08, 09, and 10 show the Bode plots of Exc(s), GEP(s), and PSS(s) from m-file for the set machine and exciter given in the system operating point where $S_{t}=0.8+j 0.6 p u$ and $\left|V_{t}\right|=1.1 p u$. The gain of the PSS(s) shown is for a $D_{P S S}$ of 6 .

It can be seen from the phase plot of GEP(s) that it has two poles near $6.5 \mathrm{~Hz}$ or $40.8 \mathrm{rad} / \mathrm{sec}$ and from the phase plot of PSS(s), we see that the desired phase characteristic of the pss 
ought to be lagging below $40.8 \mathrm{rad} / \mathrm{sec}$ and leading above that angular frequency. The phase compensation can be obtained
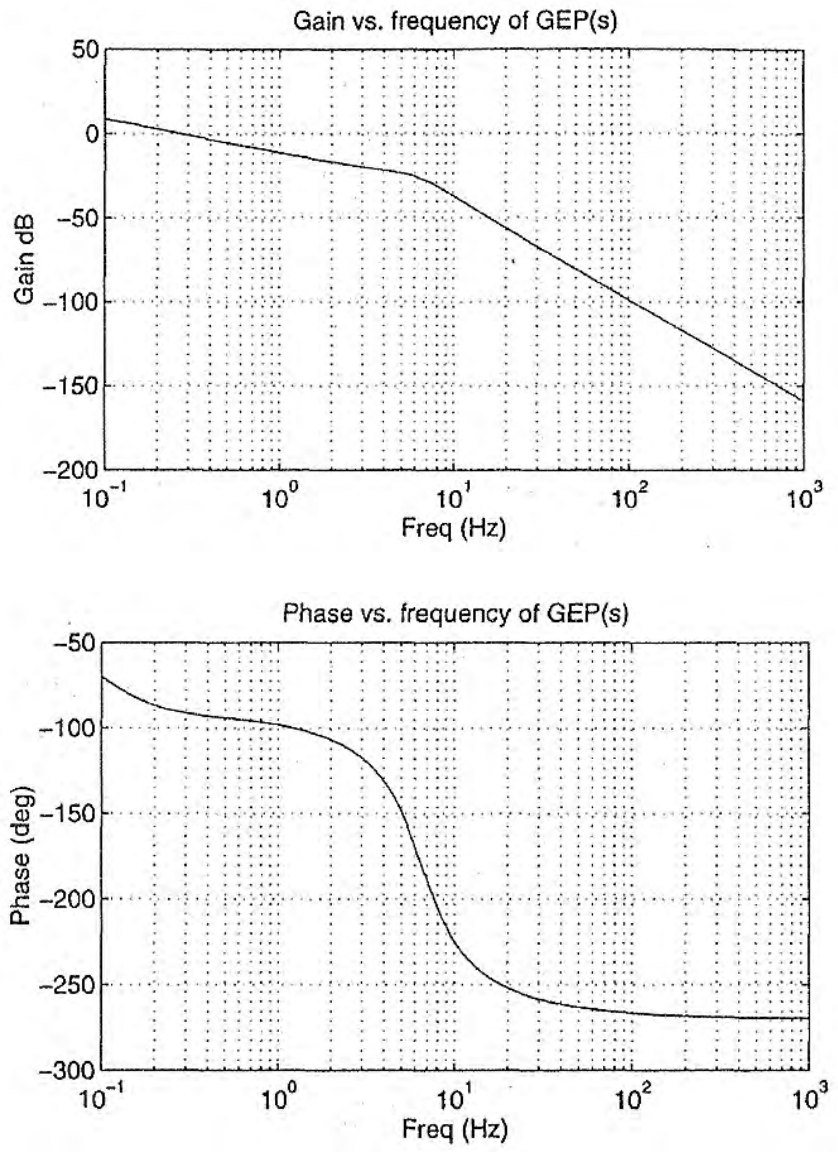

Fig. 8. Bode plot of GEP(s)

using a pair of lag-lead and lead-lag networks with zeros centered at $40.8 \mathrm{rad} / \mathrm{sec}$. Thus, picking the initial value of $T_{1}$ and $T_{3}$ to be equal to $1 / 40.8$ or 0.024 , and using roughly a $10: 1$ ratio, we can choose $T_{4}$ of the lag-lead network to be 0.24 and $T_{2}$ of the lead-lag network to be 0.002 . A sufficiently large $T_{\omega}$ of one second for the wash-out network is chosen so as not to disturb the phase compensation of the lead-lag and lag-lead networks from $1 \mathrm{~Hz}$ upwards. Too large a value of $T_{\omega}$ can result in undesirable variations of the terminal voltage along with speed for a weak or isolated condition. The preliminary value for $K_{S}$, or gain of PSS(s), is based on the desired $D_{P S S}$ of 6 . Too high a gain can result in an unstable pss loop.

A symmetrical limit of +0.1 pu or -1 pu is placed on the output of the power system stabilizer (pss). For the assumed operating condition and based on the above reasoning, we arrive at a preliminary design of the power system stabilizer (pss) with the following parameters:
The effects of the $K_{4}$ and $K_{5}$ connections in Figure 02 can be included by shifting the input of the $K_{4}$ connection to the same summing junction for $K_{5}$ and PSS, that is

$P S S(s)-K_{5} \frac{\omega_{b}}{s}-\frac{K_{4}}{\operatorname{Exc}(s)} \frac{\omega_{b}}{s}=\frac{-D_{p s s}}{G E P(s)}$

For this system, M-file, also determines the the transfer function of PSS(s) based on Equation 23, Figure 9 shows the Bode plot of PSS(s) with the effects of and connections for a of 6 and at the same operating point as that used to determine
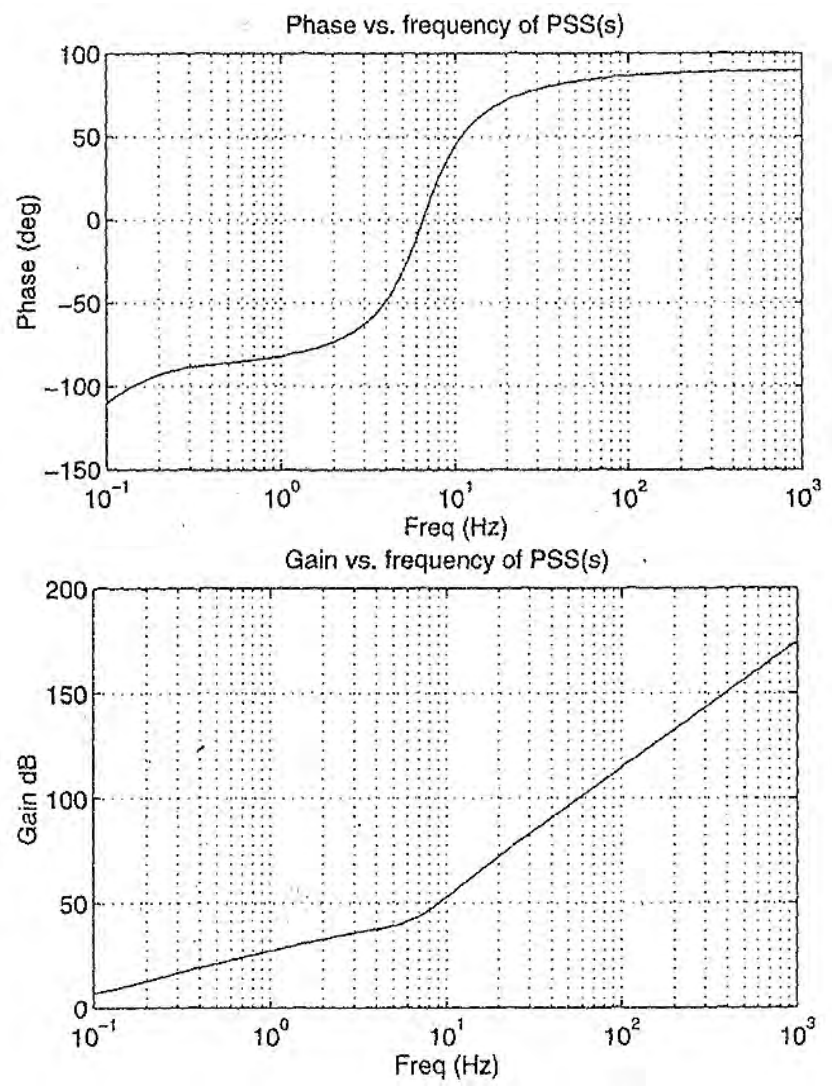

Fig. 9. Bode plot for PSS(s)

Figure 10 The difference between these two figures over the desired stabilization frequency range centered around the electromechanical oscillation frequency of $40.8 \mathrm{rad} / \mathrm{s}$ is not great.

Figure 11 shows the Bode plot of the pss transfer function with the preliminary design values. For the damping purposes, the critical part of these characteristics is that of the phase of PSS(s) about the frequency of the electromechanical oscillations that are to be damped. Figure 13 shows the root-locus of the openloop transfer function for GEPs) PSS(s). $\begin{array}{ll}\text { Wash-out network: } K_{S}=120 & T_{\omega}=1 \\ \text { Lead-lag network: } T_{1}=0.024 & T_{2}=0.002 \\ \text { Lag-lead network: } T_{3}=0.024 & T_{4}=0.24\end{array}$ 

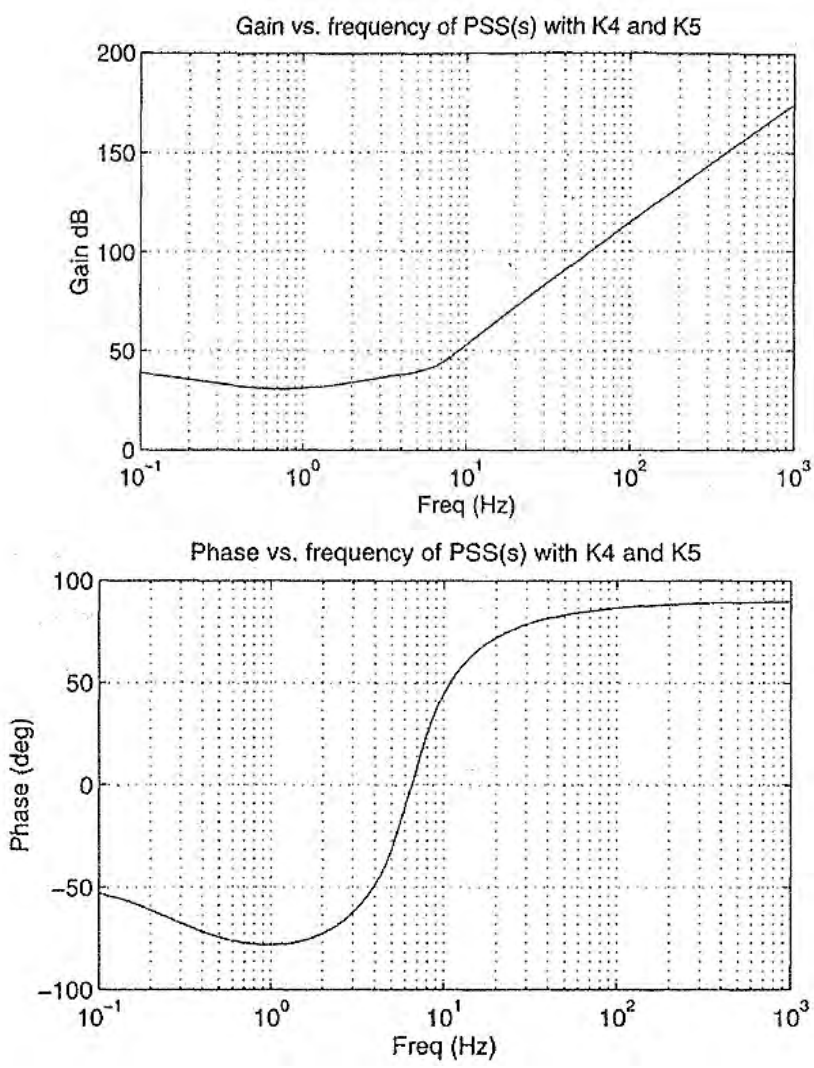

Fig. 10. Bode plot of PSS(s) with $K_{4}$ and $K_{5}$ connections
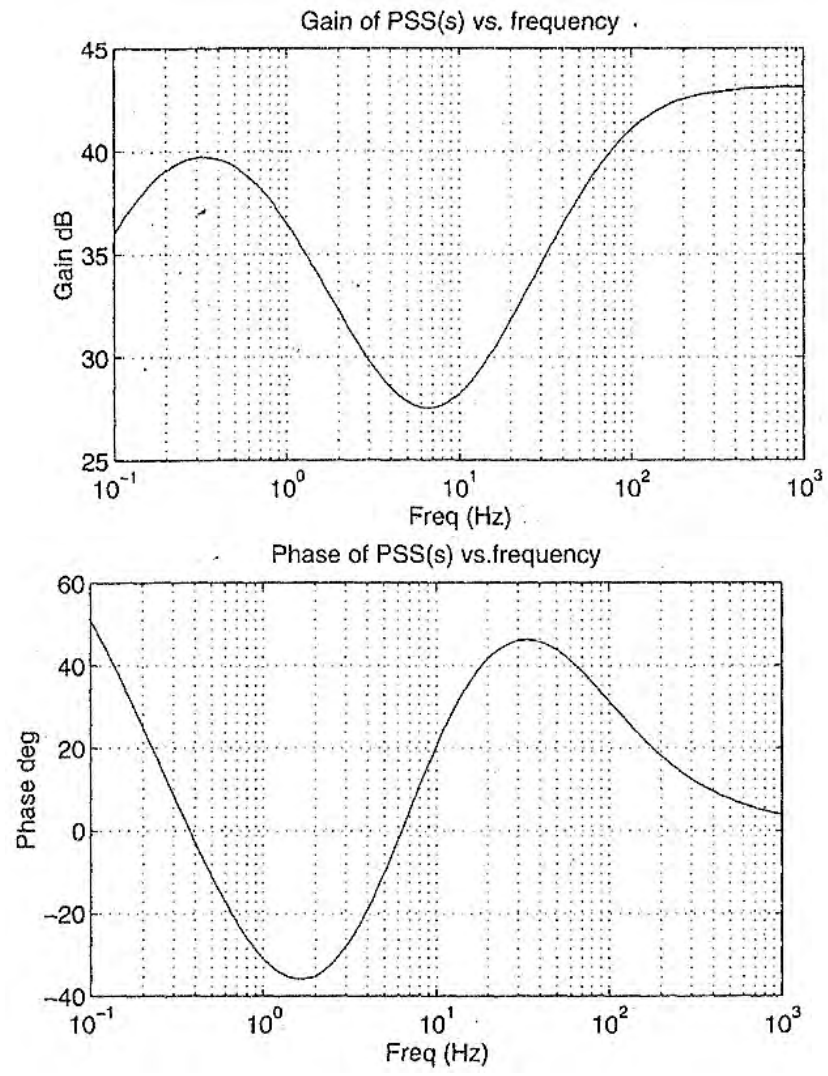

Fig. 11. Bode plot of PSS(s) with preliminary design values over a range of $K_{s}$, from 10 to 300 .

The plots in Figures 12 and 13 may be obtained using the given MATLAB m-file. As mentioned earlier, m-file also establishes the desired starting operating condition for the simulation. As given, $\mathrm{m} 4$ uses the data file, set 1 , which contains the parameters of the $9375 \mathrm{kVA}$ synchronous generator and exciter and will prompt the user to provide the impedance value of the RL line connecting the generator to the infinite bus, $r_{e}+j x_{e}$, and the voltage and delivered complex power, $V_{i}$ and $S_{i}$, at the infinite bus for it to establish the operating condition. In the design mode, $\mathrm{m}$-file will also prompt the user for the desired value of $D_{P S S}$.

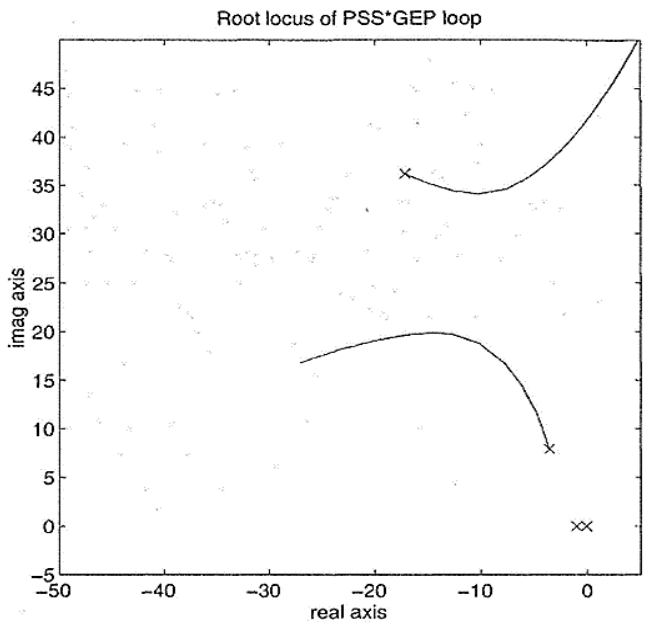

Fig. 12. Root-locus of GEP(S) * PSS(S) for $K_{5}$ up to 300

In the simulation mode, $m$-file is programmed to set array values in the repeating sequence source, DT mech, to introduce a small step torque perturbation, DT, of +0.1 pu or -0.1 pu about the chosen operating point in a fixed sequence, using the time

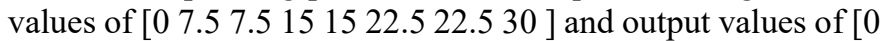
0 DT DT -DT -DT 0 0]. As given, it will also set the PSS_sw flag in simulink.

At first for a run without the power system stabilizer and after run with power system stabilizer storing the values of $\left|V_{t}\right|, \delta, P_{g e n}$ and $Q_{g e n}$ of the first run for plotting alongside those from the second run. It will plot the values of $\left|V_{t}\right|, \delta, P_{g e n}$ and $Q_{g e n}$ for each chosen operating condition. Figure 14 shows a plot of these variables for the system condition where retje $r_{e}+j x_{e}=0.027+j 0.1 p u, V_{i}=1+$ j0pu.

Use m-file to obtain a preliminary design of the pss with slip speed as the input signal using a $r_{e}+j x_{e}$ of $0.013+\mathrm{j} 0.05 p u$. Obtain the Bode plots of the transfer functions $\operatorname{GEP}(\mathrm{s})$ and PSS(s). Conduct simulation runs for the various system conditions using either the given pss design. Use $m$-file to conduct the two runs for each system condition: first without the stabilizing action of the power system stabilizer, followed by another on the same system condition but with power system stabilizer reconnected. Comment on the effects that the power system stabilizer has on the dynamic response, and on the 
observed changes with the power factor of the generator and the strength of the network connection.
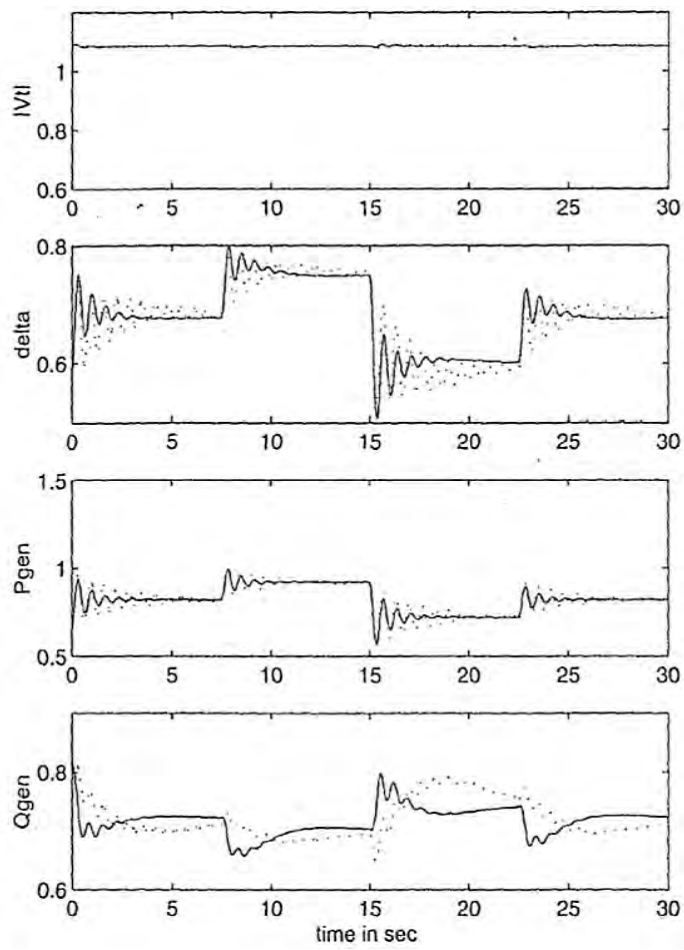

Fig. 13. System response: dotted curve for without pss and solid for with pss $r_{e}+j x_{e}=0.027+j 0.1 p u, V_{i}=1+j 0 p u$ and $S_{i}=.8+j 0.6 p u$

Using the same operating condition of a $r_{e}+j x_{e}=0.027+$ $j 0.1 p u, V_{i}=1+j 0 p u$ and $S_{i}=.8+j 0.6 p u$ that was used to obtain Figure 13 raise the value of $K_{s}$, of the pss until instability becomes apparent in simulation. ,Here value of $K_{s}$, and the nature of the instability and try to relate these with the information can extract from the root-locus plot.

\section{CONCLUSION}

This paper describes an analysis of inter area mode phenomenon in power system. The main function of a power system stabilizer is to add damping to generator rotor oscillations by controlling its excitation using auxiliary stabilizing signal. To provide damping the stabilizer must produce a component of electrical torque in phase with rotor speed deviation. The choice of control signal for power system stabilizer can be based on the following criteria. Such as the signal must be obtained from local measurements and easily synthesized. The noise content of the signal must be minimal. On the other hand complicated filters are required which can introduce their own problems. The power system stabilizer design based on a particular signal must be robust and reject noise. To avoid amplifying the noise, implies that lead compensation must be kept to a minimum problem of oscillatory instability is to provide damping for generator power oscillations. This is conveniently done by providing power system stabilizers (PSS) which are supplementary controller in the excitation system. The main function is to add damping to the generator rotor oscillations by controlling its excitation using auxiliary stabilizing signals. Simulation results (Figure 14 ) shows that when we are using power system stabilizer, the inter area oscillations have damped and system becomes stable. So, power system stabilizer is used in order to damp low interarea mode oscillations of power system. The dynamics of the system is compared with and without the presence of power system stabilizer in the system. It is clear from the simulation result that power system stabilizer is effective device to damp inter-area oscillations even in the disturbance condition. Simulation model of the synchronous generator with pss has been done in MATLAB/SIMULINK used for simulation purpose.

\section{REFERENCES}

[1] [1]. P. Kundur, Power System Stability and Control, McGraw- Hill, New York, 1994.

[2] [2]. V. Vittal, N. Bhatia, and A.A. Fouad, "Analysis of the Inter area Mode Phenomenon in Power System Following Large Disturbances," IEEE Transaction on Power System, 6(4), pp. 1515- 1521, 1991.

[3] [3]. E. V. Larsen and D. A. Swann, "Applying power system stabilizers: Part I, II, III,” IEEE Trans. Power App. Syst., vol. PAS-100, no. 6, pp. 3017-3046, June 1981.

[4] [4]. P.W. Sauer and M. A. Pai, Power System Dynamics and Stability, Prentice Hall, Upper Saddle River, New Jersey, 1998.

[5] [5]. P.Kundur, D. C. Lee, and H. M. Zein El-Din, "Power system stabilizers for thermal units: Analytical techniques and on-site validation," IEEE Trans. Power App. Syst., vol. PAS-100, no. 1, pp.8195, Jan. 1981.

[6] [6]. M. Klein, G. J. Rogers, S. Moorty, P. Kundur, "Analytical Investigation of Factors Influencing Power System Stabilizers Performances", IEEE Transaction on Energy Conversion, Vol. 7, No.3, pp. 382-390, September 1992.

[7] [7] G. Rogers, Power System Oscillations, Kluwer Academic Publishers, 2000, pp. 3, 101.

[8] [8] P. Kundur, Power System Stability and Control, McGraw-Hill, Inc., 1994, pp. 39, 706, 712, 817.

[9] [9] M Klein, G. J. Rogers, and P. Kundur, "A Fundamental Study of InterArea Oscillations in Power System”, IEEE Trans. on Power Systems, vol. 6, pp. 914-921, Feb. 1991.

[10] [10] J. S. Thorp, A. Abur, M. Begovic, J. Giri, and R. Avila-Rosales, "Gaining a wider perspective", IEEE power \& energy magazine, vol. 6, pp 43-51, 2008

[11] [11] V. Vittal, "Consequence and Impact of Electric Utility Industry Restructuring on Transient Stability and Small-Signal Stability Analysis", Proc, of the IEEE, vol. 88, pp 196-207, Feb. 2000.

[12] [12] V. Venkatasubramanian, and Y. Li, "Analysis of 1996 Western Americam Electric Blackouts", in Proc. 2004 Bulk Power System Dynamics and Control, Cortina d'Ampezzo Italy.

[13] [13] Kumar Kartikeya, Manish Kumar Singh International Journal of Innovative Research in Science, Engineering and Technology,Vol. 5, Issue 5, May 2016, ISSN(Online) : 2319-8753,ISSN (Print) : 2347-6710.

[14] [14] P. Kundur, "Power System Stability And Control", Mcgraw-Hill, 2007

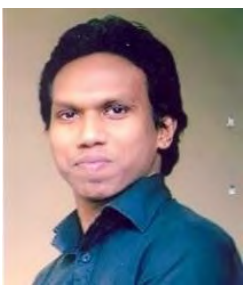

Md. Abu Hena Shatil has been serving as an assistant professor in the Department of Electrical and Electronic Engineering at American International University (AIUB). He completed his Master of science in Electrical and Electronic Engineering (MEEE) from Cardiff University,UK.

Md. Lutfur Rahman completed his Bachelor of Science in Electrical and Electronic Engineering 
(EEE) from American International University Bangladesh (AIUB) in 2017. Currently, he completed Master of Science in Electrical and Electronic Engineering from the same university. His research interest includes electronics, Telecommunications, Biomedical power system analysis, etc. 